\title{
CORRELAÇÃO ENTRE MEDIDAS PARAMÉTRICAS E NÃO-PARAMÉTRICAS DE ESTABILIDADE EM SOJA ${ }^{1}$
}

\author{
ANTONIO ORLANDO DI MAURO², VALDEMIR BERALDO CURCIOLI ${ }^{3}$, JOSÉ CARLOS MARTINS DE NÓBREGA \\ DAVID ARIOVALDO BANZATO ${ }^{4}$ e TUNEO SEDIYAMA ${ }^{5}$
}

\begin{abstract}
RESUMO - Foram avaliadas a adaptabilidade e a estabilidade de genótipos de soja (Glycine max L.) segundo a metodologia clássica de Eberhart e Russell e a estabilidade dos mesmos genótipos pela metodologia não-paramétrica de Huhn. Os experimentos foram conduzidos no delineamento em blocos casualizados, com três repetições e com 30 tratamentos (genótipos de soja), durante três anos consecutivos. As parcelas experimentais foram constituídas por quatro linhas de cultivo, espaçadas de $0,50 \mathrm{~m}$ e com densidade de 25 plantas por metro linear. Como área útil, foram tomadas as linhas centrais, eliminando-se $0,5 \mathrm{~m}$ de cada extremidade. A comparação entre as metodologias foi efetuada considerando-se o caráter produção de grãos. Verificou-se correlação de posição significativa dos postos dos genótipos, entre o desvio da regressão e as duas medidas não-paramétricas de estabilidade, porém o mesmo não foi observado entre o coeficiente de regressão e as medidas não-paramétricas $\left(\mathrm{S}_{i}^{(1)}\right.$ e $\left.\mathrm{S}_{i}^{(2)}\right)$. As medidas $\mathrm{S}_{i}^{(1)}$ e $\mathrm{S}_{\mathrm{i}}^{(2)}$ mostraram-se quase que perfeitamente correlacionadas.
\end{abstract}

Termos para indexação: Glycine max, estabilidade de genótipo.

\section{CORRELATION BETWEEN PARAMETRIC AND NON PARAMETRIC STABILITY MEASURES IN SOYBEAN}

\begin{abstract}
The adaptability and the stability of soybean (Glycine max L.)genotypes were evaluated through the classical methodology of Eberhart and Russell and the stability was also evaluated through the non-parametric methodology of Huhn. The experiments were conducted in the completely randomized blocks with three replications and with 30 treatments (soybean genotypes) during three consecutive years. The experimental plots were constituted by four rows $0.50 \mathrm{~m}$ apart and with 25 plants per meter. Data were collected in the two central rows being eliminated $0.50 \mathrm{~m}$ in each edge. To compare the two methodologies the character grain yield was considered. Positive and significative rank correlation was observed between the regression deviations and the two non-parametric stability parameters $\left(\mathrm{S}_{i}^{(1)}\right.$ and $\left.\mathrm{S}_{i}^{(2)}\right)$ for the three studied characteristcs. The parameters $\mathrm{S}_{\mathrm{i}}^{(1)}$ e $\mathrm{S}_{\mathrm{i}}^{(2)}$ were nearly perfectly correlated to each other.
\end{abstract}

Index terms: Glycine max, genotype stability.

\footnotetext{
${ }^{1}$ Aceito para publicação em 21 de maio de 1999.

${ }^{2}$ Eng. Agrôn., Prof. Adjunto, Dep. de Produção Vegetal, UNESP-Campus de Jaboticabal, CEP 14870-000 Jaboticabal, SP. E-mail: orlando@fcav.unesp.br

${ }^{3}$ Eng. Agrôn., M.Sc., Dep. de Fitotecnia, UNESP. E-mail: nobrega@fcav.unesp.br

${ }^{4}$ Eng. Agrôn., Prof. Titular, Dep. de Ciências Exatas, UNESP. E-mail: banzato@fcav.unesp.br

${ }^{5}$ Eng. Agrôn., Prof. Titular, Dep. de Fitotecnia, Universidade Federal de Viçosa, CEP 36570-000 Viçosa, MG E-mail: tsediyama@mail.ufv.br
}

\section{INTRODUÇÃO}

A soja, uma das principais leguminosas cultivadas no mundo, é sensível ao fotoperíodo e responsiva às flutuações ambientais (Mauro, 1991). Em função da crescente demanda de alimentos, a agricultura moderna necessita de cultivares que, além de rendimento médio satisfatório, manifestem conveniente sensibilidade de resposta às variações ambientais. Nessas condições, uma medida de resposta ao ambiente que exprima o conceito de estabilidade é de 
elevada importância e deve ser usada como critério na avaliação de cultivares para recomendação (Nor \& Cady, 1979).

Informações sobre a adaptabilidade relativa de um grupo de genótipos podem ser obtidas pela análise da interação genótipos com ambientes. O método paramétrico da regressão linear simples tem sido o mais utilizado e discutido na literatura. Esse procedimento relaciona o rendimento médio de cada genótipo com o índice de ambiente, provido pela resposta média dos próprios genótipos. A metodologia foi originalmente sugerida por Yates \& Cochran (1938) e, posteriormente, refinada por Finlay \& Wilkinson (1963) e por Eberhart \& Russell (1966). A maioria dos métodos utilizados para avaliar a adaptabilidade e a estabilidade dos genótipos exigem pressuposições básicas, tais como: distribuição normal dos dados, independência das variâncias e a existência de um modelo matemático associado com um delineamento experimental.

$\mathrm{Na}$ experimentação agrícola muitas vezes esses princípios básicos não são observados e, nessas situações, assume importância a existência de uma metodologia mais simples, porém efetiva na avaliação da estabilidade dos genótipos. Nassar \& Huhn (1987) apresentaram uma discussão sobre metodologias alternativas para avaliação da estabilidade fenotípica de cultivares de espécies vegetais e observaram que essas metodologias são baseadas na classificação das cultivares em cada ambiente e usam o princípio da homeostase como medida da estabilidade. Nessa metodologia, genótipos com postos similares nos diferentes ambientes são classificados como estáveis.

De acordo com Huhn (1990a), as medidas nãoparamétricas de estabilidade fenotípica, baseadas em postos, constituem uma alternativa útil e apresentam algumas vantagens sobre as medidas paramétricas, tais como: 1. redução ou prevenção de vícios causados por delineamentos; 2 . não há necessidade de pressuposições básicas; 3. há menor probabilidade de variações nas estimativas quando ocorre adição ou subtração de um ou poucos genótipos; e 4. os parâmetros de estabilidade baseados em postos são fáceis de usar e de interpretar, principalmente em programas de teste e seleção, cuja ordem dos postos dos genótipos é a informação mais essencial. Campos (1983) define o teste nãoparamétrico como sendo aquele que não especifica condições sobre os parâmetros da população da qual a amostra foi obtida. O autor ressalta que quando existem pressuposições, essas são mais brandas do que aquelas associadas com os testes paramétricos e, com isso, o emprego da estatística não-paramétrica constitui-se valiosa alternativa de trabalho dada a sua simplicidade e versatilidade.

Considerando-se que em um programa de melhoramento de soja, quando os genótipos já se encontram próximos da homozigose, a disponibilidade de sementes muitas vezes não permite o estabelecimento de repetições nos ambientes em que o melhorista trabalha e que informações sobre a estabilidade dos materiais em seleção pode se constituir num critério seletivo, o presente trabalho objetivou verificar a efetividade do método não-paramétrico de Huhn (1990a) em relação ao método clássico de Eberhart \& Russell (1966) na determinação da estabilidade de genótipos de soja.

\section{MATERIAL E MÉTODOS}

Os dados utilizados para as comparações entre o método paramétrico de Eberhart \& Russell (1966) e o método não-paramétrico de Huhn (1990a) foram obtidos em ensaios de avaliação de genótipos de soja, conduzidos no delineamento em blocos ao acaso, com três repetições e por três anos consecutivos. As parcelas foram constituídas por quatro linhas de cultivo, com $5 \mathrm{~m}$ de comprimento, com 25 plantas por metro linear e, como área útil, foram consideradas as linhas centrais, eliminando-se $0,5 \mathrm{~m}$ de cada extremidade. O caráter analisado foi a produção de grãos, com teor de umidade de $13 \%$ e fornecido em gramas/ parcela. Os tratamentos foram constituídos por 30 genótipos de soja, a saber: UFV 87-69, Sucupira, UFV 87-279, UVF 87-587, UFV 85-558, Doko, UFV 87-321, UFV 87-54, UFV 84-028A, UFV 87-427, UFV 87-313, UFV 87-357, UFV 85-552, UFV 82-506, UFV 86-649, UFV 84-127, UFV 87-272, UFV 87-265, UFV 87-448, UFV 87-109, UFV 87-214, UFV 87-249, UFV 87-217, UFV 87-291, UFV 86-613, UFV 86-650, UFV 86-627, UFV 87-692, UFV 86-604 e UFV 87-48.

Os dados foram inicialmente submetidos a uma análise conjunta para verificação da significância da interação genótipos por ambientes. Após esses procedimentos preliminares, foram efetuadas análises segundo as metodologias 
de Eberhart \& Russell (1966) e de Huhn (1990a). Os demais testes e procedimentos estatísticos foram efetuados de acordo com as sugestões de Snedecor \& Cochran (1989) e de Campos (1983).

\section{Análise segundo Eberhart e Russell}

$\mathrm{Na}$ análise de adaptabilidade e de estabilidade segundo a metodologia paramétrica, cada ano de cultivo foi considerado como um ambiente, perfazendo um total de três ambientes estudados. A regressão de cada cultivar, em relação ao índice de ambiente e os quadrados dos desvios dessa regressão forneceram estimativas dos parâmetros de estabilidade, que foram definidos pelo seguinte modelo matemático:

$Y_{\mathrm{ij}}=\mu_{\mathrm{i}}+\beta_{\mathrm{i}} \mathrm{I}_{\mathrm{j}}+\delta_{\mathrm{ij}}+\varepsilon_{\mathrm{ij}}$, em que:

$\mathrm{Y}_{\mathrm{ij}}=$ média das repetições do i-ésimo genótipo no j-ésimo ambiente;

$\mu_{\mathrm{i}}=$ média do i-ésimo genótipo em todos os ambientes;

$\beta_{\mathrm{i}}=$ coeficiente de regressão linear para o i-ésimo genótipo;

$\mathrm{I}_{\mathrm{j}}=$ índice de ambiente, fornecido pela diferença entre a

média do j-ésimo ambiente e a média geral de todos os genótipos em todos os ambientes;

$\delta_{\mathrm{ij}}=$ desvio da regressão linear do i-ésimo genótipo no j-ésimo ambiente e

$\varepsilon_{\mathrm{ij}}=$ erro aleatório associado à observação $\mathrm{Y}_{\mathrm{ij}}$.

Os parâmetros indicativos da adaptabilidade e da estabilidade foram determinados como segue:

Média geral do i-ésimo genótipo $=\mu_{\mathrm{i}}=(1 / \mathrm{a}) \sum_{\mathrm{j}} \mathrm{Y}_{\mathrm{ij}}$, em que:

$\mathrm{a}=$ número de ambientes.

Coeficiente de regressão linear associado ao i-ésimo genótipo $=\beta_{\mathrm{i}}=\sum_{\mathrm{j}} \mathrm{Y}_{\mathrm{ij}} \mathrm{I}_{\mathrm{j}} / \sum_{\mathrm{j}} \mathrm{I}_{\mathrm{j}}^{2}$, em que:

$\mathrm{I}_{\mathrm{j}}=(1 / \mathrm{g}) \sum_{\mathrm{i}} \mathrm{Y}_{\mathrm{ij}}-(1 / \mathrm{ga}) \sum_{\mathrm{i}} \sum_{\mathrm{j}} \mathrm{Y}_{\mathrm{ij}}$ e

$\mathrm{V}(\mathrm{b})=\left(1 / \Sigma \mathrm{I}_{\mathrm{j}}^{2}\right)(\mathrm{QME} / \mathrm{r})$,

$\mathrm{g}=$ número de genótipos;

$\mathrm{r}=$ número de repetições e

$\mathrm{QME}=$ quadrado médio do erro.

Desvio da regressão linear $=\mathrm{s}^{2}{ }_{\mathrm{di}}=(\mathrm{QM}$ desvio $\mathrm{da}$ regressão - QME)/r, em que:

$\mathrm{QM}=$ quadrado médio e

$\mathrm{s}_{\mathrm{di}}^{2}=$ estimador do componente de variância $\sigma_{\mathrm{di}}^{2}$.

Coeficiente de determinação $\left(\mathrm{R}^{2}\right)=$

SQ regressão linear $\left(\mathrm{a} / \mathrm{g}_{\mathrm{i}}\right) / \mathrm{SQ}$ total $\left(\mathrm{a} / \mathrm{g}_{\mathrm{i}}\right)$.

Para testar a hipótese de que o coeficiente de regressão não difere da unidade, foi utilizado o teste $t$, dado por: $\mathrm{t}=(\mathrm{b}-1) /[\mathrm{V}(\mathrm{b})]^{1 / 2}$. A significância dos desvios da regres- são $\left(\mathrm{s}^{2}{ }_{\mathrm{di}}\right)$ foi verificada pelo teste $\mathrm{F}$ aproximado, dado por: $\mathrm{F}=\left(\sum_{\mathrm{j}}\left(\delta_{\mathrm{ij}}^{2} / \mathrm{a}-2\right) / \mathrm{QME}\right.$, em que:

$\mathrm{a}=$ número de ambientes.

Segundo Mauro (1991), é muito importante a correta interpretação dos parâmetros de adaptabilidade e de estabilidade e, para esse autor, o coeficiente da regressão $\left(b_{i}\right)$ fornece um indicativo do grau de adaptabilidade do genótipo i. Nessas condições, se $b_{i}=1$, o genótipo associado tem desempenho diretamente proporcional à melhoria do ambiente, se relacionado com uma média alta indica elevada adaptabilidade a todos os ambientes, e se baixa, o genótipo tem baixa adaptabilidade aos vários ambientes; se $b_{i}>1$ o genótipo possui uma elevada capacidade de responder vantajosamente às melhorias ambientais, e se $\mathrm{b}_{\mathrm{i}}<1$ o genótipo relacionado é particularmente adaptado a ambientes desfavoráveis. Por outro lado, os desvios da linearidade $\left(\mathrm{s}^{2}{ }_{\mathrm{di}}\right)$ permitem o estabelecimento de inferências sobre a estabilidade genotípica. Com isso, quando o desvio é não-significativo o genótipo associado tem comportamento estável e previsível; quando os desvios são significativos, o genótipo relacionado tem comportamento instável e imprevisível. Outro parâmetro útil nesses estudos é o coeficiente de determinação $\left(\mathrm{R}^{2}\right)$, que expressa a adequação do modelo utilizado.

\section{Análise segundo Huhn (1990a)}

Nas análises pelo método não-paramétrico foi utilizada uma tabela de dupla entrada com $\mathrm{K}$ genótipos e $\mathrm{N}$ ambientes, denotando-se o valor fenotípico do i-ésimo genótipo no j-ésimo ambiente como $\mathrm{x}_{\mathrm{ij}}$, onde $\mathrm{i}=1,2, \ldots, \mathrm{Ke}$ $\mathrm{j}=1,2, \ldots, \mathrm{N}$. Dentro de cada ambiente $\mathrm{j}$ foi calculado o valor corrigido, $\mathrm{x}_{\mathrm{ij}}^{*}=\mathrm{x}_{\mathrm{ij}}-\left(\overline{\mathrm{x}}_{\mathrm{i}} \cdot-\overline{\mathrm{x}} ..\right)$ conforme sugestão de Nassar \& Huhn (1987). Foi atribuído ao menor valor o posto 1 , e ao maior, o posto $\mathrm{K}$, onde $\overline{\mathrm{X}}_{\mathrm{i}}$.é a média marginal do genótipo i e $\overline{\mathrm{X}}$..é a média global. Considerando-se $r_{i j}$ como o posto do genótipo i no ambiente $j$, o genótipo i é estável nos ambientes se seus postos são similares e a estabilidade máxima ocorre com posto semelhante em todos os ambientes.

As duas medidas de estabilidade de Huhn (1990a) são expressas como segue:

$$
\begin{aligned}
& \mathrm{S}_{\mathrm{i}}^{(1)}=2 \sum_{\mathrm{j}=1}^{\mathrm{N}-1} \sum_{\mathrm{j}^{\prime}=\mathrm{j}+1}^{\mathrm{N}}\left|\mathrm{r}_{\mathrm{ij}}-\mathrm{r}_{\mathrm{ij}}\right| /[\mathrm{N}(\mathrm{N}-1)] \mathrm{e} \\
& \mathrm{S}_{\mathrm{i}}^{(2)}=\sum_{\mathrm{j}=1}^{\mathrm{N}}\left(\mathrm{r}_{\mathrm{ij}}-\overline{\mathrm{r}}_{\mathrm{i}} \cdot\right)^{2} /(\mathrm{N}-1), \text { em que: }
\end{aligned}
$$


$\overline{\mathrm{r}}_{\mathrm{i}}=$ é a média dos postos em todos os ambientes para o i-ésimo genótipo,

$\mathrm{S}_{\mathrm{i}}^{(1)}=$ é a média das diferenças absolutas dos postos de um genótipo i nos $\mathrm{N}$ ambientes,

$\mathrm{S}_{\mathrm{i}}^{(2)}=$ é a variância desses postos.

Os testes de significância para esses dois parâmetros foram desenvolvidos por Huhn \& Nassar (1989) e, inicialmente, a hipótese da nulidade de que todos os genótipos apresentam estabilidades similares foi testada somando-se os valores de, $\mathrm{S}_{\mathrm{i}}^{(\mathrm{m})}$ onde $\mathrm{m}=1,2$ para todos os genótipos, como segue:

$$
\sum_{i=1}^{K} Z_{i}^{(m)}=\sum_{i=1}^{K}\left[S_{i}^{(m)}-E\left(S_{i}^{(m)}\right)\right]^{2} /\left(S_{i}^{(m)}\right)
$$

De acordo com Huhn \& Nassar (1989) esse teste tem distribuição de qui-quadrado, com número de graus de liberdade igual ao número de genótipos. Sob a hipótese da nulidade, as esperanças $E\left(S_{i}^{(m)}\right)$ e as variâncias $\operatorname{Var}\left(\mathrm{S}_{\mathrm{i}}^{(\mathrm{m})}\right)$ podem ser expressas como segue:

$$
\begin{aligned}
& \mathrm{E}\left(\mathrm{S}_{\mathrm{i}}^{(1)}\right)=\frac{\mathrm{K}^{2}-1}{3 \mathrm{~K}} \\
& \mathrm{E}\left(\mathrm{S}_{\mathrm{i}}^{(2)}\right)=\frac{\mathrm{K}^{2}-1}{12} \\
& \operatorname{Var}\left(\mathrm{S}_{\mathrm{i}}^{(1)}\right)=\frac{\left(\mathrm{K}^{2}-1\right)\left[\left(\mathrm{K}^{2}-4\right)(\mathrm{N}+3)+30\right]}{45 \mathrm{~K}^{2} \mathrm{~N}(\mathrm{~N}-1)} \\
& \operatorname{Var}\left(\mathrm{S}_{\mathrm{i}}^{(2)}\right)=\frac{\left(\mathrm{K}^{2}-1\right)\left[2\left(\mathrm{~K}^{2}-4\right)(\mathrm{N}-1)+5\left(\mathrm{~K}^{2}-1\right)\right]}{360 \mathrm{~N}(\mathrm{~N}-1)}
\end{aligned}
$$

Nas análises de estabilidade, quando a hipótese da nulidade foi rejeitada, examinou-se a estabilidade de um genótipo específico pelo teste $Z_{\mathrm{i}}^{(\mathrm{m})}$, que também possui distribuição aproximada de qui-quadrado, com 1 grau de liberdade (Nassar \& Huhn, 1987).

\section{Comparação dos métodos pelo coeficiente de correla- ção de Spearman}

Os parâmetros estimadores da adaptabilidade e da estabilidade dos genótipos foram comparados pelo coeficiente de correlação de posição de Spearman $\left(\mathrm{r}_{\mathrm{pp}}\right)$, sendo observadas as sugestões de Campos (1983). Adotaram-se os seguintes procedimentos: 1 . as estimativas dos parâmetros p e p' foram colocadas em ordem decrescente; 2. foi obtida a diferença $d_{i}$ entre o posto do genótipo $i$ no parâmetro $\mathrm{p}$ e no parâmetro p' e 3 . foi calculado o coeficiente de correlação de posição entre os parâmetros p e p', mediante emprego da expressão: $\mathrm{r}_{\mathrm{pp}},=1-\left[\left(6 \Sigma \mathrm{d}_{\mathrm{i}}^{2}\right) /\left(\mathrm{J}^{3}-\mathrm{J}\right)\right]$. A hipótese $\mathrm{H}_{0}: \mathrm{r}_{\mathrm{pp}},=0$ foi verificada pelo teste $\mathrm{t}$, com $\mathrm{J}-2$ graus de liberdade e dado por: $\mathrm{t}=\mathrm{r}_{\mathrm{pp}},\left[(\mathrm{J}-2) /\left(1-\mathrm{r}_{\mathrm{pp}}^{2}\right)\right]^{1 / 2}$, em que $\mathrm{J}=$ número de ambientes.

\section{RESULTADOS E DISCUSSÃO}

\section{Análise segundo Eberhart e Russell}

Na Tabela 1 encontram-se os resultados da análise de variância conjunta, para o caráter produção de grãos, podendo-se constatar que não foi verificado efeito significativo apenas para genótipos; para ambientes e para a interação genótipos x ambientes verificou-se significância a $1 \%$ de probabilidade. $\mathrm{O}$ coeficiente de variação foi de baixa magnitude $(11,20 \%)$ e sugere boa precisão para o experimento. Os resultados obtidos sugerem a existência de diferenças significativas entre os ambientes considerados e que os genótipos comportaram-se diferencialmente nos ambientes.

Os resultados da análise de variância segundo o modelo paramétrico encontram-se na Tabela 2 . Houve efeito significativo, a $1 \%$ de probabilidade, para a fonte de variação entre ambientes dentro de genótipos. Esse resultado era esperado, porquanto esse efeito corresponde à soma dos efeitos ambientais e da interação genótipo x ambiente, com posterior desdobramento em ambiente linear, interação linear e desvios combinados, o que permite o estabelecimento de inferências acerca dos efeitos lineares e não-lineares relacionados com a adaptabilidade e com a estabilidade dos genótipos.

$\mathrm{Na}$ mesma tabela pode-se observar que a interação genótipos $\mathrm{x}$ ambiente (linear) revelou-se

TABELA 1. Resultados da análise de variância conjunta para todos os ambientes, referente à característica produção de grãos.

\begin{tabular}{lcc}
\hline Fonte de variação & $\begin{array}{c}\text { Grau de } \\
\text { liberdade }\end{array}$ & $\begin{array}{c}\text { Quadrado } \\
\text { médio }\end{array}$ \\
\hline Blocos/ambiente & 6 & -- \\
Genótipo & 29 & $44540,9221^{\mathrm{ns}}$ \\
Ambiente & 2 & $9326351,2148^{* *}$ \\
Genótipo x ambiente & 58 & $35836,3719^{* *}$ \\
Resíduo & 174 & 5208,1292 \\
\hline C.V. (\%) & & 11,20 \\
${ }_{\text {ns }}$ e $^{* *}$ Não-significativo e significativo a $1 \%$ de probabilidade pelo teste $\mathrm{F}$.
\end{tabular}


TABELA 2. Resultados da análise de variância segundo o modelo paramétrico de Eberhart e Russell (1966), para a característica produção de grãos.

\begin{tabular}{|c|c|c|}
\hline Fonte de variação & Graus de liberdade & Quadrado médio \\
\hline Genótipos & 29 & $14846,9740^{\mathrm{ns}}$ \\
\hline Ambientes & 2 & $3108783,7383^{* *}$ \\
\hline Genótipos $\mathrm{x}$ ambiente & 58 & $11945,4553^{* *}$ \\
\hline Ambientes/genótipos & 60 & $115173,4000^{* *}$ \\
\hline Ambientes linear & 1 & $217567,4765^{* *}$ \\
\hline Genótipos x ambientes (linear) & 29 & $18654,2645^{* *}$ \\
\hline Desvios combinados & 30 & $5062,6951 * *$ \\
\hline Desv. UFV 87-69 & 1 & $14436,4489 * *$ \\
\hline Desv. Sucupira & 1 & $18,4541^{\mathrm{ns}}$ \\
\hline Desv. UFV 87-279 & 1 & $3496,4650^{\mathrm{ns}}$ \\
\hline Desv. UFV 87-587 & 1 & $2687,1625^{\mathrm{ns}}$ \\
\hline Desv. UFV 85-558 & 1 & $1986,6486^{\mathrm{ns}}$ \\
\hline Desv. Doko & 1 & $3795,8781^{\mathrm{ns}}$ \\
\hline Desv. UFV 87-321 & 1 & $2427,4092^{\mathrm{ns}}$ \\
\hline Desv. UFV 87-54 & 1 & $720,3739^{\text {ns }}$ \\
\hline Desv. UFV 84-028A & 1 & $13218,6125 * *$ \\
\hline Desv. UFV 87-427 & 1 & $612,7878^{\text {ns }}$ \\
\hline Desv. UFV 87-313 & 1 & $4750,2328^{\mathrm{ns}}$ \\
\hline Desv. UFV 87-357 & 1 & $10008,6957^{*}$ \\
\hline Desv. UFV 85-552 & 1 & $6417,7216^{\mathrm{ns}}$ \\
\hline Desv. UFV 82-506 & 1 & $15378,9658 * *$ \\
\hline Desv. UFV 86-649 & 1 & $2970,8910^{\mathrm{ns}}$ \\
\hline Desv. UFV 84-127 & 1 & $9510,8197 *$ \\
\hline Desv. UFV 87-272 & 1 & $590,7410^{\mathrm{ns}}$ \\
\hline Desv. UFV 87-265 & 1 & $3298,8228^{\mathrm{ns}}$ \\
\hline Desv. UFV 87-448 & 1 & $17943,8243 * *$ \\
\hline Desv. UFV 87-109 & 1 & $279,7393^{\text {ns }}$ \\
\hline Desv. UFV 87-214 & 1 & $390,1046^{\mathrm{ns}}$ \\
\hline Desv. UFV 87-249 & 1 & $153,4089^{\mathrm{ns}}$ \\
\hline Desv. UFV 87-217 & 1 & $11489,2665^{*}$ \\
\hline Desv. UFV 87-291 & 1 & $113,5185^{\mathrm{ns}}$ \\
\hline Desv. UFV 86-613 & 1 & $5304,9093^{\mathrm{ns}}$ \\
\hline Desv. UFV 86-650 & 1 & $2130,2293^{\mathrm{ns}}$ \\
\hline Desv. UFV 86-627 & 1 & $5691,7399^{\mathrm{ns}}$ \\
\hline Desv. UFV 87-692 & 1 & $11002,9325^{*}$ \\
\hline Desv. UFV 86-604 & 1 & $796,0982^{\text {ns }}$ \\
\hline Desv. UFV 87-48 & 1 & $239,9496^{\mathrm{ns}}$ \\
\hline Resíduo & 174 & 1736,0431 \\
\hline
\end{tabular}

significativa a $1 \%$ de probabilidade, evidenciando a existência de diferenças genéticas entre os genótipos com relação aos seus comportamentos lineares, quando submetidos a variações ambientais. Os efeitos lineares de ambiente também se revelaram significativos a $1 \%$ de probabilidade, o que sugere que a característica em estudo apresentou reatividade frente aos ambientes considerados. Os desvios combinados mostraram-se altamente significativos, sugerindo que os componentes linear e não-linear da estabilidade encontram-se envolvidos na performance fenotípica dos genótipos nos ambientes considerados. 
Na Tabela 3 encontram-se os valores relativos aos parâmetros de adaptabilidade e de estabilidade de Eberhart e Russell, podendo-se verificar que Sucupira e UFV 87-109 apresentaram coeficientes de regressão significativos e superiores à unidade, pelo teste $\mathrm{t}$, a $5 \%$ de probabilidade. Tais resultados sugerem que esses genótipos possuem grande capacidade de explorar vantajosamente os estímulos ambientais favoráveis. As médias associadas a esses genótipos também sugerem que os mesmos possuem boa adaptação aos ambientes estudados. Além disso, os genótipos apresentaram desvios da regressão não-significativos e coeficientes de determinação de alta magnitude, mostrando serem estáveis e previsíveis.

Os demais genótipos apresentaram coeficientes de regressão não-significativos pelo teste t, o que os caracteriza como tendo desempenho diretamente proporcional à melhoria do ambiente. Os genótipos UFV 87-69, UFV 87-279, Doko, UFV 87-321, UFV 87-313, UFV 82-506, UFV 86-649, UFV 84-127, UFV 87-265, UFV 87-249, UFV 86-613, UFV 86-627 e UFV 87-48 apresentaram produção superior à média geral, sugerindo que possuem elevada adaptabilidade a todos os ambientes. Os genótipos restantes apresentaram produção abaixo da média geral, o que os caracteriza como de baixa adaptabilidade aos ambientes considerados.

$\mathrm{Na}$ mesma tabela observa-se ainda que os genótipos UFV 87-69, UFV 84-028A, UFV 87-357, UFV 82-506, UFV 84-127, UFV 87-448, UFV 87-217 e UFV 87-692 apresentaram desvios da regressão significativos pelo teste $\mathrm{F}$, sugerindo instabilidade e imprevisibilidade face às alterações ambientais. Os demais genótipos estudados apresentaram desvios da regressão não-significativos, o que indica estabilidade e previsibilidade comportamental.

\section{Análise segundo Huhn (1990a)}

Os resultados relativos à análise de estabilidade fenotípica segundo o método não-paramétrico encontram-se na Tabela 4. Pode-se verificar que o parâmetro $Z_{i}^{(1)}$ apresentou valor de $\sum_{i=1}^{30} Z_{i}^{(2)}=36,68$, enquanto que $Z_{i}^{(2)}$ apresentou valor de $\sum_{i=1}^{30} Z_{i}^{(2)}=48,11$; sendo este último superior ao encontrado pelo teste qui-quadrado (á ${ }_{0,05 ; 30}=43,77$ ). Os resultados do teste $\mathrm{S}_{\mathrm{i}}^{(1)}$, que avalia a média das diferenças absolutas dos postos, sugerem que alguns genótipos são estáveis. Por sua vez, o teste $\mathrm{S}_{\mathrm{i}}^{(2)}$, que avalia a variância dos postos dos genótipos, sugere a presença de genótipos instáveis e, nessa situação, é conveniente analisar individualmente cada genótipo.

Analisando-se os valores individuais de $Z_{\mathrm{i}}^{(2)}$ de cada genótipo, constata-se que UFV 87-279 $\left(\mathrm{Z}_{\mathrm{i}}^{(2)}=8,8067\right)$ mostrou-se altamente instável em relação aos demais genótipos, porquanto apresentou valor de $Z_{i}^{(2)}$ superior ao valor crítico do teste quiquadrado $\left(a^{2}{ }_{0,01 ; 1}=6,63\right)$. Porém, quando se considera os valores individuais $Z_{\mathrm{i}}^{(2)}$ e o nível de significância de $5 \%$ ( á $\left._{0,05 ; 1}=3,84\right)$, verifica-se na mesma tabela que os genótipos UFV 87-54, UFV 84-028A, UFV 87-109 e UFV 87-692 também apresentaram valores superiores ao valor crítico do teste qui-quadrado, caracterizando-se como instáveis. Dessa forma, pelo método não-paramétrico de Huhn (1990a), cinco genótipos entre os 30 estudados podem ser classificados como instáveis.

\section{Comparação dos métodos pelo coeficiente de correla- ção de Spearman}

A Tabela 5 apresenta as correlações de posição de Spearman entre os parâmetros de adaptabilidade e estabilidade dos métodos paramétrico e nãoparamétrico, para o caráter produção de grãos. Como esperado, o coeficiente de regressão $\left(b_{i}\right)$ de Eberhart \& Russell (1966), que mede a adaptabilidade fenotípica, não se correlacionou com as medidas nãoparamétricas de Huhn (1990a). Pode-se, entretanto, constatar correlação positiva e significativa entre os desvios da regressão $\left(\mathrm{s}^{2}{ }_{\mathrm{di}}\right)$, que medem a estabilidade fenotípica no modelo paramétrico, e os valores $S_{i}^{(1)}$ e $S_{i}^{(2)}(0,5067$ e 0,4605 , respectivamente), que também medem o mesmo parâmetro no modelo não-paramétrico. Deve-se destacar que as medidas não-paramétricas $\mathrm{S}_{\mathrm{i}}^{(1)}$ e $\mathrm{S}_{\mathrm{i}}^{(2)}$ revelaram-se perfeitamente correlacionadas. 
TABELA 3. Produtividade média $\left(m_{\mathrm{i}}\right)$, estimativa do coeficiente de regressão $\left(b_{i}\right)$, dos desvios da regressão $\left(s^{2} d_{i}\right)$ e coeficiente de determinação $\left(R^{2}\right)$ dos 30 genótipos de soja estudados pelo método paramétrico de Eberhart \& Russell (1966).

\begin{tabular}{|c|c|c|c|c|}
\hline Genótipos & $\mathrm{m}_{\mathrm{i}}$ & $b_{i}$ & $\mathrm{~s}^{2} \mathrm{~d}_{\mathrm{i}}$ & $\mathrm{R}^{2}$ \\
\hline UFV 87-69 & 682 & $0,8956^{\mathrm{ns}}$ & $12700,4059 * *$ & 0,9281 \\
\hline Sucupira & 691 & $1,3301^{*}$ & $-1717,5890^{\mathrm{ns}}$ & 1,0000 \\
\hline UFV 87-279 & 837 & $1,6259^{\mathrm{ns}}$ & $1760,4219^{\mathrm{ns}}$ & 0,9937 \\
\hline UFV 87-587 & 562 & $0,7819^{\mathrm{ns}}$ & $951,1194^{\mathrm{ns}}$ & 0,9792 \\
\hline UFV 85-558 & 625 & $0,8086^{\mathrm{ns}}$ & $250,6055^{\mathrm{ns}}$ & 0,9856 \\
\hline Doko & 661 & $0,8512^{\mathrm{ns}}$ & $2059,8350^{\mathrm{ns}}$ & 0,9753 \\
\hline UFV 87-321 & 719 & $0,7856^{\mathrm{ns}}$ & $691,3661^{\mathrm{ns}}$ & 0,9814 \\
\hline UFV 87-54 & 567 & $0,5442^{\mathrm{ns}}$ & $-1015,6692^{\mathrm{ns}}$ & 0,9884 \\
\hline UFV 84028A & 525 & $0,6712^{\mathrm{ns}}$ & $11482,5694 * *$ & 0,8760 \\
\hline UFV $87-427$ & 632 & $1,1687^{\mathrm{ns}}$ & $-1123,2553^{\mathrm{ns}}$ & 0,9978 \\
\hline UFV 87-313 & 699 & $1,2208^{\mathrm{ns}}$ & $3014,1898^{\text {ns }}$ & 0,9849 \\
\hline UFV 87-357 & 522 & $0,7055^{\mathrm{ns}}$ & $8272,6527^{*}$ & 0,9115 \\
\hline UFV 85-552 & 518 & $0,7419^{\mathrm{ns}}$ & $4681,6786^{\mathrm{ns}}$ & 0,9467 \\
\hline UFV 82-506 & 761 & $1,3307^{\mathrm{ns}}$ & $13642,9227 * *$ & 0,9598 \\
\hline UFV 86-649 & 678 & $1,1153^{\mathrm{ns}}$ & $1234,8479^{\mathrm{ns}}$ & 0,9886 \\
\hline UFV 84-127 & 645 & $0,7706^{\mathrm{ns}}$ & $7774,776^{*}$ & 0,9283 \\
\hline UFV 87-272 & 632 & $0,9678^{\mathrm{ns}}$ & $-1145,3020^{\mathrm{ns}}$ & 0,9970 \\
\hline UFV 87-265 & 666 & $1,2032^{\mathrm{ns}}$ & $1562,7797^{\mathrm{ns}}$ & 0,9891 \\
\hline UFV 87-448 & 636 & $1,2556^{\mathrm{ns}}$ & $16207,7812 * *$ & 0,9479 \\
\hline UFV 87-109 & 678 & $1,4740 *$ & $-1456,3038^{\mathrm{ns}}$ & 0,9994 \\
\hline UFV 87-214 & 596 & $0,6805^{\mathrm{ns}}$ & $-1345,9385^{\mathrm{ns}}$ & 0,9960 \\
\hline UFV 87-249 & 728 & $1,2467^{\mathrm{ns}}$ & $-1582,6342^{\mathrm{ns}}$ & 0,9995 \\
\hline UFV 87-217 & 605 & $1,2916^{\mathrm{ns}}$ & $9753,2234 *$ & 0,9678 \\
\hline UFV 87-291 & 626 & $0,8536^{\mathrm{ns}}$ & $-1622,5246^{\mathrm{ns}}$ & 0,9992 \\
\hline UFV 86-613 & 683 & $0,9534^{\mathrm{ns}}$ & $3568,8662^{\mathrm{ns}}$ & 0,9726 \\
\hline UFV 86-650 & 608 & $1,1405^{\mathrm{ns}}$ & $394,1862^{\text {ns }}$ & 0,9922 \\
\hline UFV 86-627 & 649 & $1,4654^{\mathrm{ns}}$ & $3955,6968^{\mathrm{ns}}$ & 0,9874 \\
\hline UFV 87-692 & 609 & $0,4890^{\text {ns }}$ & $9266,8894 *$ & 0,8183 \\
\hline UFV 86-604 & 595 & $0,7948^{\mathrm{ns}}$ & $-939,9449^{\text {ns }}$ & 0,9940 \\
\hline UFV $87-48$ & 677 & $0,7851^{\mathrm{ns}}$ & $-1496,0935^{\mathrm{ns}}$ & 0,9981 \\
\hline População & 644 & - & - & - \\
\hline
\end{tabular}

ns, * e ** Não-significativo e significativo a $5 \%$ e a $1 \%$ de probabilidade, respectivamente, pelo teste $\mathrm{F}$.

Normalmente, coeficientes de correlação entre 0,40 e 0,75 são considerados de média magnitude, e os resultados apresentados relacionam-se com a performance conjunta de todos os genótipos dentro dos métodos avaliados. Nessas condições, as duas metodologias evidenciaram alguma correspondência na avaliação da estabilidade fenotípica e o método não-paramétrico pode ser empregado para avaliação da estabilidade fenotípica de soja em determinadas circunstâncias.
Outros autores (Huhn, 1990b; Piepho \& Lotito, 1992; Pelá, 1997), trabalhando com outras culturas, reportaram resultados semelhantes.

De acordo com Mariotti et al. (1976), a adaptabilidade refere-se à capacidade de os genótipos aproveitarem vantajosamente os estímulos ambientais, e estabilidade relaciona-se com a capacidade de os genótipos apresentarem comportamento previsível em função dos estímulos ambientais. Salientam Minor \& Berlato (1977) que a análise de estabilidade é muito importante para melhor caracterizar as culti- 
TABELA 4. Resultados da análise de estabilidade pelo método não-paramétrico de Huhn (1990a).

\begin{tabular}{|c|c|c|c|c|c|c|}
\hline Genótipo & $\begin{array}{c}\text { Produção } \\
\text { média }(\mathrm{g} / \mathrm{p})\end{array}$ & $\begin{array}{l}\text { Posto } \\
\text { médio }\end{array}$ & $\mathrm{S}_{\mathrm{i}}^{(1)}$ & $\mathrm{Z}_{1}^{(1)}$ & $\mathrm{S}_{\mathrm{i}}^{(2)}$ & $Z_{1}^{(2)}$ \\
\hline UFV 87-69 & 682 & 15,6667 & 14,6667 & 1,0929 & 121,333 & 0,6407 \\
\hline Sucupira & 691 & 13,3333 & 14,0000 & 0,8036 & 140,333 & 1,2727 \\
\hline UFV 87-279 & 837 & 12,0000 & 19,3333 & 4,3611 & 247,000 & 8,8067 \\
\hline UFV 87-587 & 562 & 17,3333 & 19,3333 & 0,5586 & 100,333 & 0,1921 \\
\hline UFV 85-558 & 625 & 16,3333 & 8,0000 & 0,1976 & 44,333 & 0,2782 \\
\hline Doko & 661 & 17,0000 & 9,3333 & 0,0215 & 61,000 & 0,0576 \\
\hline UFV 87-321 & 719 & 16,3333 & 10,0000 & 0,0000 & 60,333 & 0,0632 \\
\hline UFV 87-54 & 567 & 18,3333 & 17,3333 & 0,6940 & 202,333 & 4,8282 \\
\hline UFV 84-028A & 525 & 14,6667 & 18,0000 & 3,2053 & 192,333 & 4,1001 \\
\hline UFV 87-427 & 632 & 14,0000 & 6,6667 & 0,5512 & 28,000 & 0,6546 \\
\hline UFV 87-313 & 699 & 14,0000 & 12,6667 & 0,3581 & 97,000 & 0,1450 \\
\hline UFV 87-357 & 522 & 16,0000 & 16,0000 & 1,8047 & 147,000 & 1,5433 \\
\hline UFV 85-552 & 518 & 16,3333 & 14,6667 & 1,0929 & 122,333 & 0,6686 \\
\hline UFV 82-506 & 761 & 15,6667 & 16,0000 & 1,8047 & 152,333 & 1,7824 \\
\hline UFV 86-649 & 678 & 14,6667 & 8,0000 & 0,1976 & 44,333 & 0,2782 \\
\hline UFV 84-127 & 645 & 15,6667 & 14,0000 & 0,8036 & 134,333 & 1,0499 \\
\hline UFV 87-272 & 632 & 16,6667 & 4,6667 & 1,4147 & 12,333 & 1,1648 \\
\hline UFV 87-265 & 666 & 13,6667 & 11,3333 & 0,0903 & 76,333 & 0,0006 \\
\hline UFV 87-448 & 636 & 16,3333 & 14,6667 & 1,0929 & 154,333 & 1,8757 \\
\hline UFV 87-109 & 678 & 13,0000 & 16,6667 & 2,2272 & 193,000 & 4,1468 \\
\hline UFV 87-214 & 596 & 17,6667 & 14,6667 & 1,0929 & 142,333 & 1,3517 \\
\hline UFV 87-249 & 728 & 14,0000 & 10,0000 & 0,0000 & 75,000 & 0,0000 \\
\hline UFV 87-217 & 605 & 16,6667 & 14,6667 & 1,0929 & 142,333 & 1,3517 \\
\hline UFV 87-291 & 626 & 17,3333 & 4,0000 & 1,7913 & 9,333 & 1,2792 \\
\hline UFV 86-613 & 683 & 15,3333 & 9,3333 & 0,0215 & 49,333 & 0,1946 \\
\hline UFV 86-650 & 608 & 14,0000 & 7,3333 & 0,3522 & 31,000 & 0,5736 \\
\hline UFV 86-627 & 649 & 13,3333 & 18,0000 & 3,2053 & 186,333 & 3,6918 \\
\hline UFV 87-692 & 609 & 15,6667 & 19,3333 & 4,3611 & 210,333 & 5,4536 \\
\hline UFV 86-604 & 595 & 17,0000 & 8,0000 & 0,1976 & 36,000 & 0,4504 \\
\hline UFV $87-48$ & 677 & 17,0000 & 8,0000 & 0,1976 & 48,000 & 0,2155 \\
\hline Total & & & & 36,6846 & & 48,1135 \\
\hline \multicolumn{7}{|c|}{ Teste estático } \\
\hline & $\mathrm{E}\left(\mathrm{S}_{1}{ }^{1}\right)$ & $\mathrm{E}\left(\mathrm{S}_{1}^{2}\right)$ & $\operatorname{Var}\left(\mathrm{S}_{1}{ }^{1}\right)$ & $\operatorname{Var}\left(\mathrm{S}_{1}^{2}\right)$ & $\alpha^{2} Z_{1}, Z_{2}$ & $\alpha^{2} \Sigma Z_{1}, Z_{2}$ \\
\hline & 9,9888 & 74,9167 & 20,0222 & 3362,51 & 6,63 & 43,7730 \\
\hline & \multicolumn{6}{|c|}{$\alpha_{0,05 ; 1}^{2}=3,84$} \\
\hline
\end{tabular}

vares e permitir a identificação das melhores condições edafoclimáticas para cada cultivar. Com isso, num programa de melhoramento assume grande importância a estabilidade fenotípica, posto que ao melhorista de plantas interessa o desenvolvimento de genótipos com comportamento previsível, o que 
TABELA 5. Comparação dos parâmetros de adaptabilidade e de estabilidade dos modelos paramétricos e não-paramétricos, pelo coeficiente de correlação de Spearman.

\begin{tabular}{cccc}
\hline & $\mathrm{b}_{\mathrm{i}}$ & $\mathrm{s}^{2} \mathrm{~d}_{\mathrm{i}}$ & $S_{i}^{(2)}$ \\
\hline$S_{i}^{(1)}$ & $-0,048^{\mathrm{ns}}$ & $0,5067^{* *}$ & $0,9380^{* *}$ \\
$S_{i}^{(2)}$ & $0,038^{\mathrm{ns}}$ & $0,4605^{*}$ & \\
\hline
\end{tabular}

ns, * e ** Não-significativo e significativo a $5 \%$ e a $1 \%$ de probabilidade, respectivamente, pelo teste $t$.

facilita os processos de recomendação de cultivares para as diversas regiões produtoras.

\section{CONCLUSÕES}

1. A metodologia paramétrica de Eberhart \& Russell (1966) evidencia maior sensibilidade na detecção de diferenças na estabilidade dos genótipos estudados.

2. A metodologia não-paramétrica de Huhn (1990a) caracteriza-se pela simplicidade e facilidade na interpretação dos resultados.

3. Os dois métodos apresentam alguma divergência na detecção do grau de estabilidade de alguns genótipos, e as medidas não-paramétricas apresentam correlação de média magnitude com o desvio da regressão.

4. O método não-paramétrico pode ser empregado na cultura da soja quando não se tem a observância das pressuposições estatísticas básicas ou ausência de delineamentos experimentais nos ambientes estudados.

5. As medidas não-paramétricas de estabilidade correlacionam-se quase perfeitamente, revelando alto grau de correspondência na detecção dos genótipos estáveis.

\section{REFERÊNCIAS}

CAMPOS, H. Estatística experimental não paramétrica. Piracicaba : ESALQ, 1983.349p.
EBERHART, S.A.; RUSSELL, W.A. Stability parameters for comparing varieties. Crop Science, Madison, v.6, n.1, p.36-40, 1966.

FINLAY, K.W.; WILKINSON, G.N. The analysis of adaptation in a plant breeding programme. Australian Journal of Agricultural Research, Collingwood, v.14, n.6, p.742-754, 1963.

HUHN, M. Nonparametric measures of phenotipic stability. Part I: Theory. Euphytica, Dordrecht, v.47, n.3, p.189-194, 1990a.

HUHN, M. Nonparametric measures of phenotipic stability. Part II: Applications. Euphytica, Dordrecht, v.47, n.3, p.195-201, 1990b.

HUHN, M.; NASSAR, R. On tests of significance for nonparametric measures of phenotipic stability. Biometrics, Dordrecht, v.45, p.997-1000, 1989.

MARIOTTI, J.A.; OYARZABAL, E.S.; OSA, J.M.; BULACIO, A.N.R.; ALMADA, G.H. Analisis de estabilidad de genótipos de cana de azucar. I. Interacciones dentro de una localidad experimental. Revista Agronomica del Noroeste Argentino, Tucumán, v.13, n.1/4, p.105-127, 1976.

MAURO, A.O. Adaptabilidade, estabilidade e ganho genético com o processo seletivo em soja, em Ponta Porã, MS. Viçosa : Universidade Federal de Viçosa, Imprensa Universitária, 1991. 192p. Tese de Doutorado.

MINOR, H.C.; BERLATO, M.A. Comportamento de seis cultivares de soja em 42 ambientes do Rio Grande do Sul. Agronomia Sulriograndense, Porto Alegre, v.13, n.1, p.83-92, 1977.

NASSAR, R.; HUHN, M. Studies on estimation of phenotipic stability: Tests of significance for nonparametric measures of phenotipic stability. Biometrics, Dordrecht, v.43, n.1, p.45-53, 1987.

NOR, K.M.; CADY, F.B. Methodology for identifying wide adaptability in crops. Agronomy Journal, Madison, v.71, n.4, p.556-559, 1979.

PELÁ, L.A. Estabilidade fenotípica de cultivares de milho híbrido por método não paramétrico.

Pesq. agropec. bras., Brasília, v.35, n.4, p.687-696, abr. 2000 
Jaboticabal : UNESP-Faculdade de Ciências Agrárias e Veterinárias, 1997. 86p. Dissertação de Mestrado.

PIEPHO, H.P.; LOTITO, S. Rank correlation among parametric and non parametric measures of phenotypic stability. Euphytica, Dordrecht, v.64, p.221-225, 1992.
SNEDECOR, G.W.; COCHRAN, W.G. Statistical methods. 8.ed. Ames : Iowa State University, 1989. $503 \mathrm{p}$.

YATES, F.; COCHRAN, W.G. The analysis of groups of experiments. Journal of Agricultural Science, Cambridge, v.28, p.556-580, 1938. 